\title{
Risk Factors for Positional Vertigo and the Impact of Vertigo on Daily Life: The Korean National Health and Nutrition Examination Survey
}

\author{
Moo Kyun Park ${ }^{1 *}$, Doh Young Lee ${ }^{2 *}$, and Young Ho Kim² \\ ${ }^{1}$ Department of Otorhinolaryngology-Head and Neck Surgery, Seoul National University College of Medicine, Seoul, Korea \\ ${ }^{2}$ Department of Otorhinolaryngology-Head and Neck Surgery, Seoul National University Boramae Medical Center, \\ Seoul National University College of Medicine, Seoul, Korea
}

\begin{abstract}
Received April 4, 2018
Revised May 28, 2018

Accepted June 1, 2018
\end{abstract}

\section{Address for correspondence \\ Young Ho Kim, MD, PhD \\ Department of Otolaryngology-Head \\ and Neck Surgery, \\ Seoul National University \\ Boramae Medical Center, \\ Seoul National University \\ College of Medicine, \\ 20 Boramae-ro 5-gil, Dongjak-gu, \\ Seoul 07061, Korea \\ Tel $+82-2-870-2442$ \\ Fax +82-2-870-3863 \\ E-mail yhkiment@gmail.com \\ *These authors contributed equally to this work.}

Background and Objectives: This study aimed to analyze risk factors for positional vertigo (PV) and the influence thereof on daily life and subjective quality-of-life (QoL). Subject and Methods: A cross-sectional study was conducted using data of the 2010 Korea National Health and Nutrition Examination Survey. The study population consisted of 1,274 individuals aged $>40$ years for whom complete dizziness-related data were available. Blood and urine tests were performed, and nutritional intake, QoL, and subjective health status were measured using a questionnaire. The associations between PV and blood/urine test data and nutritional intake were evaluated via multiple logistic regression analysis. Results: A history of PV within the previous year was reported by 98 individuals (7.7\%). Advanced age and female gender were both significantly associated with such a history. Serum hemoglobin, creatinine, and triglyceride levels correlated significantly with a history of PV. Carotene, vitamin A, and vitamin B2 intakes were significantly lower in individuals with PV. Multivariate analysis revealed that only age was significantly associated with a history of $P V(p=0.003)$. Although subjective health status score was not significantly lower in subjects with PV, subjective impairments in mobility, self-care, the performance of usual activities, and anxiety/depression were more prominent in individuals with PV. A fall history and limitations of activity were also significantly higher in individuals with PV $(p<0.001$ and $p=0.003$, respectively) Conclusions: Age was a risk factor for PV, which affected most QoL parameters, fall frequency, and the performance of normal activities.

J Audiol Otol 2019;23(1):8-14

\section{Introduction}

Positional vertigo (PV) is a common cause of vertigo in older adults. Among them, benign paroxysmal positional vertigo (BPPV) is the most common form of PV, which impairs quality-of-life $(\mathrm{QoL})$ and increases the risk of falls $[1,2]$. The average annual incidence of $\mathrm{PV}$ ranges from 11 to 64 per 100,000 and peaks in subjects aged $50-70$ years [3]. PV is associated with vestibular dysfunction or imbalance, abnormal otolith positioning (in BPPV), and cerebral dysfunc-

This is an Open Access article distributed under the terms of the Creative Commons Attribution Non-Commercial License (https://creativecommons.org/licenses/by-nc/4.0/) which permits unrestricted non-commercial use, distribution, and reproduction in any medium, provided the original work is properly cited. tion [4]. Patients with heart disease or abnormal blood test results frequently report dizziness associated with positional change, which can be differentiated from PV [2]. PV sometimes becomes chronic and seriously affects daily life [5]. To date, no preventative strategy has been described. It is important to define risk factors for particular groups. Previous studies reported that those lacking regular physical activity and nutritional imbalances may be associated with metabolic and circulatory changes triggering various symptoms including dizziness and even PV [6]. However, risk factors for PV have not been well evaluated. Most PV is relatively shortlived and is diagnosed without evaluation of blood test data or nutritional status $[2,4]$.

Here, we sought to define risk factors for PV in South Ko- 
rea. We evaluated several blood test parameters and nutritional status based on data obtained by the 2010 Korea National Health and Nutrition Examination Survey (KNHANES). We also assessed associations between PV and QoL and fall risk.

\section{Subjects and Methods}

\section{Study population and data collection}

We analyzed data from the fifth KNHANES performed in 2010. The KNHANES is an ongoing nationwide survey of non-institutionalized South Korean subjects and features high-level standardization and quality control. All data were collected and managed by the Korean Centers for Disease Control and Prevention. Each year, an expert panel selected 192 districts for evaluation, and 20 households in each district were sampled. The data are representative of those of the entire Korean population. Statisticians weighted the data by adjusting the post-stratification and non-response rates, and defined extreme values. Thus, the data are representative of the civilian, non-institutionalized South Korean population. Stratified, multistage clustered sampling was performed based on the national census data of the National Statistical Office.

The study followed all relevant tenets of the Declaration of Helsinki. Prior written informed consent was obtained from all participants. The study protocol was reviewed and approved by the Ethics Committee of the Korea University Medical Center.

\section{PV diagnosis}

Participants were asked: "Have you experienced dizziness or imbalance in the past 12 months?" Those who answered positively were then asked: "Have you experienced severe vertigo during the past 12 months when you rotate your head in the supine position or when you sit up in bed or lie down in the morning?" (this is the definition of PV). Subjects were also asked: "Have you ever fallen unexpectedly in the past 12 months?"

To assess vestibular function, the modified Romberg test of balance during standing on firm and compliant surfaces was performed. Participants were asked to stand on a firm surface with their feet $10 \mathrm{~cm}$ apart for at least $15 \mathrm{~s}$ in the position assumed for computerized dynamic posturography, with the arms crossed, without bending the knees or moving the body. The eyes were first open (condition 1) and then closed (condition 2). Next, we assessed the effect of eliminating somatosensory input on postural stability. We repeated the above procedure but with all subjects standing on an 18-cm-thick, mediumdensity foam pad (made of polyurethane; density $22 \mathrm{~kg} / \mathrm{m}^{3}$ ) with the eyes open (condition 3 ) and then closed (condition 4) for at least $20 \mathrm{~s}$. Individual balance was graded as pass or fail.
A fail was recorded if a subject moved the feet, unfolded the hands, opened the eyes, or required operator intervention to maintain balance. The foam pad performance when the eyes are closed (condition 4) yields information on the contribution of the vestibular system to postural stability; visual input is eliminated and somatosensory inputs masked. Therefore, the modified Romberg test was considered failed when a subject passed test conditions $1-3$ but not condition 4 , indicating vestibular dysfunction.

All tympanic membranes (both right and left) were examined for signs of prior ear disease by trained otologists and subjects were categorized into three groups: normal, abnormal, and not examinable. The pure-tone audiometric thresholds at $0.5,1,2,3,4$, and $6 \mathrm{kHz}$ were measured in both ears using an automated audiometer (GSI SA-203, Entomed Diagnostics AB, Malmö, Sweden) with all subjects in soundproof booths. Unilateral or bilateral hearing loss (HL) was defined as an HL $>25 \mathrm{~dB}$ when the air-conduction hearing thresholds at $0.5,1,2$, and $3 \mathrm{kHz}$ in one or both ears were averaged.

\section{Detection of risk factors}

Age, gender, body mass index (BMI), and smoking history were categorized as personal risk factors. Patients were grouped by 10 -year age intervals: $40-50,51-60,61-70$, and $71-80$ years. Current and past smoking histories were recorded, as were daily cigarette numbers. Parental smoking habits were also noted.

Blood tests included the red blood cell count; the hemoglobin $\mathrm{Hb}$ level; the white blood cell count; and the levels of blood urea nitrogen, creatinine $(\mathrm{Cr})$, glucose, 2.2 glutamate-oxaloacetate transaminase, 2.1 glutamate-pyruvate transaminase, triglyceride (TG), total cholesterol, high-density lipoprotein, low-density lipoprotein, and alkaline phosphatase. Urine parameters recorded included the $\mathrm{pH}$ and specific gravity, and protein, glucose, ketone, blood, bilinogen, cotinine, and $\mathrm{Cr}$ levels.

The intakes of 112 foods most frequently consumed by Koreans were recorded using a semi-quantitative food-frequency questionnaire. Subjects replied how often, on average, they had consumed each of the foods listed and the usual serving sizes consumed during the study year. For each food, intake frequency was recorded as: "I do not eat that food," "once per month," "2-3 times per month," "once per week," "2-4 times per week," "5-6 times per week," "one a day," "twice a day," and "3 times a day." Nutritional parameters evaluated included protein, fat, saturated fatty acid, monounsaturated fatty acid, polyunsaturated fatty acid, cholesterol, carbohydrate, dietary fiber, calcium, phosphorus, iron, sodium, potassium, vitamin A, carotene, retinol, vitamin B1, vitamin B2, vitamin B3, and vitamin $\mathrm{C}$ intakes. 


\section{Effects of PV on quality-of-life}

Health-related QoL was assessed using the EuroQol instrument, which has following parameters: A health-status descriptive component (EuroQoL 5-dimension, EQ-5D) and an EQ visual analog scale. The EQ-5D records the levels of problems by self-reporting in the spheres of mobility, self-care, engagement in usual activities, pain/discomfort, and anxiety/depression $[7,8]$. Each parameter is assessed by posing a single question with three possible responses (extreme problems, some problems, and no problem). Additionally, the EQ-5D questionnaire features a visual analog scale ranging from 0 (worst imaginable health state) to 100 (best imaginable health state), allowing respondents to subjectively assess their health.

\section{Statistical analysis}

The sample weights for all participants, reflecting the complex probability of inclusion in the survey, were applied to all statistical data. Adjusted odds ratios (aORs) were calculated after applying these weighted values. The associations between blood/urine test data and nutritional status, and BPPV, were subjected to multiple logistic regression analysis. Continuous outcomes were compared between groups using the independent-samples t-test. Dichotomous outcomes were analyzed using the chi-squared test. aORs with $95 \%$ confidence intervals (CIs) were calculated, and a $p<0.05$ was considered to indicate statistical significance. All analyses were performed with SPSS ver. 20.0 software (IBM Corp., Armonk, NY, USA).

\section{Results}

\section{Study population}

Of the 4,126 original enrollees, individuals meeting the following historical criteria were excluded: 1) stroke, heart/lung/ kidney/thyroid disease, diabetes, and depression associated with dizziness caused by a particular disease or medication $(\mathrm{n}=$ $2,423) ; 2)$ a malignant tumor $(\mathrm{n}=244) ; 3)$ anemia $(\mathrm{n}=526)$; 4) chronic otitis media or HL [either self-reported or evident on tympanic membrane examinations with/without unilateral or bilateral HL on the hearing test $(\mathrm{n}=269)$ ]; and 5) other dizziness disorders ( $\mathrm{n}=74)$ (Fig. 1).

Thus, a total of 1,221 individuals with a mean age of 51.1 years (range, $40-83$ years) and a male-to-female ratio of 1.18:1 were included. The PV prevalence in the cohort was $8.0 \%$ ( $\mathrm{n}=$ 98). Among individuals with PV, 86 had PV histories and 12 currently had PV.

\section{Risk factors for PV}

Age and gender were significantly associated with $\mathrm{PV}$ on univariate analysis ( $p=0.012$ and $p=0.002$, respectively) (Table 1). Older age and female gender were associated with higher PV prevalence, but BMI did not differ significantly between subjects with and without PV. The PV levels among subjects reporting dizziness did not differ significantly by age group (37.5\% in those aged $40-50,32.6 \%$ in those aged $51-60$, $33.3 \%$ in those aged $61-70$, and $35.3 \%$ those aged over 70 years; $p=0.956$ ). Current smoking status and a history of alcohol consumption did not correlate significantly with a PV history ( $p=0.543$ and $p=0.487$, respectively). In terms of laboratory data, the serum $\mathrm{Hb}, \mathrm{Cr}$, and TG levels were significantly associated with $\mathrm{PV}(p=0.002, p=0.041$, and $p=0.039$, respectively) in the univariate analysis (Table 1). No urine test parameter was significantly associated with PV. Lower intakes of vitamin A, carotene, and vitamin B2 were significantly associated with $\mathrm{PV}(p=0.007, p=0.007$, and $p=0.020$, respectively) (Table 2).

Multivariate analysis revealed that advancing age (aOR, $1.029 ; 95 \%$ CI, $1.006-1.053)$ was the only significant risk factor for PV $(p=0.003)$ (Table 3).

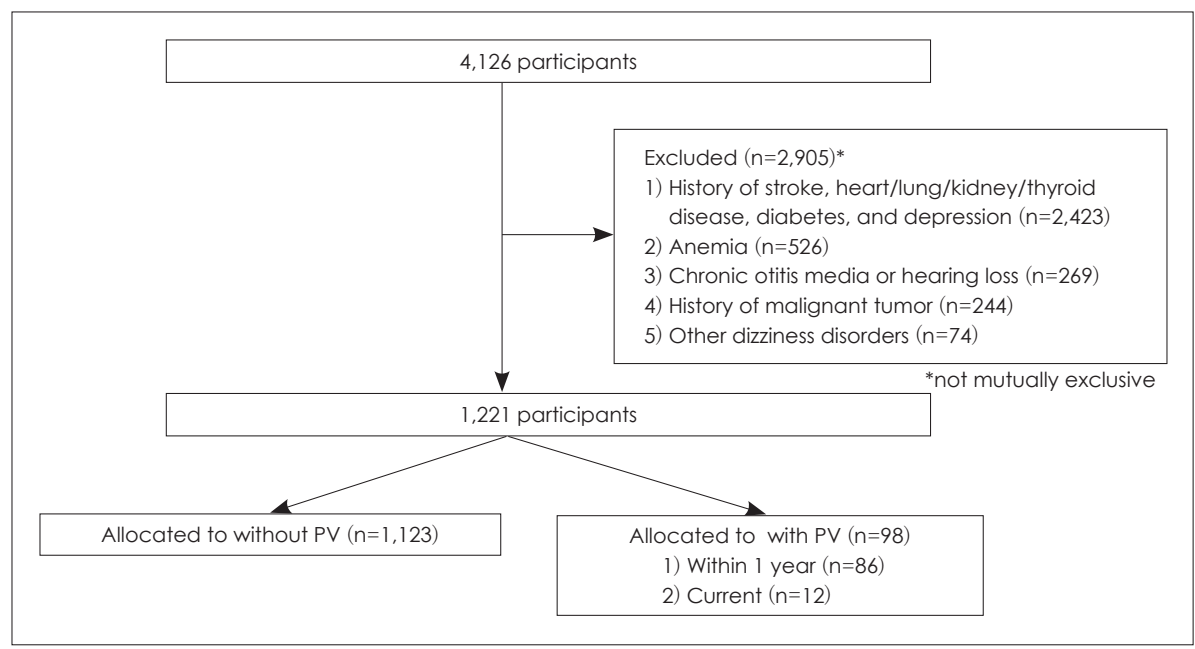

Fig. 1. Study population: a flow diagram of exclusions. PV: positional vertigo.

10 J Audiol Otol 2019;23(1):8-14 
Table 1. Risk factors for PV: epidemiological parameters and blood and urine test markers

\begin{tabular}{|c|c|c|c|}
\hline & $\mathrm{PV}(+)(\mathrm{n}=98)$ & $P \vee(-)(n=1,123)$ & p-value \\
\hline Age (years) & $54.4 \pm 10.7$ & $51.1 \pm 9.1$ & $0.012^{*}$ \\
\hline Gender ratio (M:F) & $0.63: 1$ & $1.18: 1$ & $0.002^{*}$ \\
\hline BMI $\left(\mathrm{kg} / \mathrm{cm}^{2}\right)$ & $23.6 \pm 2.9$ & $23.9 \pm 2.9$ & 0.606 \\
\hline \multicolumn{4}{|l|}{ Blood test results } \\
\hline $\mathrm{Hb}(\mathrm{g} / \mathrm{dL})$ & $14.0 \pm 1.2$ & $14.4 \pm 1.3$ & $0.002^{*}$ \\
\hline WBC $\left(\times 10^{9} / \mathrm{L}\right)$ & $5.9 \pm 1.6$ & $6.1 \pm 1.6$ & 0.312 \\
\hline Platelets $\left(\times 10^{9} / \mathrm{L}\right)$ & $249.3 \pm 52.8$ & $250.1 \pm 54.4$ & 0.893 \\
\hline BUN (mg/dL) & $13.7 \pm 3.7$ & $14.2 \pm 3.7$ & 0.216 \\
\hline $\mathrm{Cr}(\mathrm{mg} / \mathrm{dL})$ & $0.8 \pm 0.2$ & $0.8 \pm 0.2$ & $0.041^{*}$ \\
\hline Glucose (mg/dL) & $95.0 \pm 15.0$ & $95.2 \pm 15.2$ & 0.896 \\
\hline GOT (IU/L) & $22.5 \pm 8.9$ & $22.7 \pm 10.0$ & 0.830 \\
\hline GPT (IU/L) & $21.5 \pm 19.1$ & $22.1 \pm 15.0$ & 0.770 \\
\hline Y-GTP (IU/L) & $33.8 \pm 50.1$ & $38.1 \pm 50.1$ & 0.416 \\
\hline Total cholesterol (mg/dL) & $199.6 \pm 37.6$ & $197.7 \pm 36.3$ & 0.642 \\
\hline LDL (mg/dL) & $123.0 \pm 24.6$ & $122.1 \pm 32.5$ & 0.864 \\
\hline $\mathrm{HDL}(\mathrm{mg} / \mathrm{dL})$ & $48.5 \pm 11.8$ & $47.8 \pm 10.3$ & 0.594 \\
\hline TG $(\mathrm{mg} / \mathrm{dL})$ & $118.3 \pm 61.5$ & $144.1 \pm 122.7$ & $0.039^{*}$ \\
\hline Vitamin D & $18.8 \pm 6.7$ & $18.8 \pm 6.6$ & 0.987 \\
\hline ALP (IU/L) & $231.2 \pm 70.8$ & $221.3 \pm 63.8$ & 0.187 \\
\hline PTH & $61.7 \pm 18.1$ & $65.1 \pm 21.3$ & 0.172 \\
\hline \multicolumn{4}{|l|}{ Urine test results } \\
\hline $\mathrm{pH}$ & $5.8 \pm 0.9$ & $5.7 \pm 0.8$ & 0.327 \\
\hline SG & $1.02 \pm 0.01$ & $1.02 \pm 0.01$ & 0.050 \\
\hline Protein (mg/dL) & $0.04 \pm 0.21$ & $0.06 \pm 0.24$ & 0.592 \\
\hline Ketones (mg/dL) & $0.14 \pm 0.57$ & $0.10 \pm 0.34$ & 0.328 \\
\hline Glucose (mg/dL) & $0.01 \pm 0.10$ & $0.04 \pm 0.34$ & 0.053 \\
\hline Bilirubin (mg/dL) & $0.24 \pm 0.65$ & $0.22 \pm 0.63$ & 0.814 \\
\hline Blood (mg/dL) & $0.80 \pm 1.12$ & $0.68 \pm 1.08$ & 0.316 \\
\hline Bilinogen (mg/dL) & $0.01 \pm 0.10$ & $0.00 \pm 0.07$ & 0.574 \\
\hline Cotinine $(\mathrm{ng} / \mathrm{mL})$ & $673.8 \pm 1179.2$ & $544.1 \pm 963.0$ & 0.604 \\
\hline $\mathrm{Cr}(\mathrm{mg} / \mathrm{dL})$ & $131.9 \pm 82.5$ & $138.4 \pm 69.0$ & 0.394 \\
\hline
\end{tabular}

*p<0.05. PV: positional vertigo, BMl: body mass index, Hb: hemoglobin, WBC: white blood cell, BUN: blood urea nitrogen, Cr: creatinine, GOT: 2.2 glutamate-oxaloacetate transaminase, GPT: 2.1 glutamate-pyruvate transaminase, TG: triglyceride, HDL: high-density lipoprotein, LDL: low-density lipoprotein, ALP: alkaline phosphatase, PTH: parathyroid hormone, SG: specific gravity

\section{Impact of PV on daily life}

The extent of functional impairment as reflected in the EQ$5 \mathrm{D}$ responses was significantly greater in individuals with $\mathrm{PV}$ (Fig. 2). Among all PV subjects, 20.4, 7.1, 15.3, and 11.2\% reported impairments in mobility, self-care, the performance of usual activities, and anxiety/depression, respectively. All values were significantly higher than those of individuals lacking PV $(p<0.001, p=0.001, p<0.001$, and $p=0.003$, respectively). However, the EQ visual analog scale data revealed that PV did not significantly impact subjective health status. Subjects with and without PV had similar visual ana$\log$ scale scores $(76.6 \pm 98.9$ vs. $77.4 \pm 15.2$, respectively, $p=0.799$ ).

A history of falls was significantly more common in sub- jects with BPPV ( $8.1 \%$ vs. $0.2 \%, p<0.001)$. Objective limitations on daily activity (e.g., absence from work) were more prevalent in PV individuals (12.2\% vs. 5.7\%) $(p=0.003)$ (Fig. 3).

\section{Discussion}

We found that older adults, especially older than 70 years old, without any underlying disease were at higher risk of PV compromising many aspects of the QoL, including mobility, self-care, engagement in usual activities, and anxiety/depression. Moreover, the risk of falls and the extent of limitations on activity were significantly more prevalent in those with than in those without PV.

PV reflects either peripheral or central vestibular dysfunc- 
Table 2. Nutritional risk factors for PV

\begin{tabular}{lccc}
\hline & $\mathrm{PV}(+)(\mathrm{n}=98)$ & $\mathrm{PV}(-)(\mathrm{n}=1,123)$ & $\mathrm{p}$-value \\
\hline Water intake $(\mathrm{g})$ & $1,011.9 \pm 565.2$ & $1,125.9 \pm 695.7$ & 0.079 \\
Food intake $(\mathrm{g})$ & $1,481.6 \pm 693.8$ & $1,630.0 \pm 836.9$ & 0.062 \\
Carbohydrate intake $(\mathrm{g})$ & $333.4 \pm 130.0$ & $343.1 \pm 122.8$ & 0.502 \\
Protein intake $(\mathrm{g})$ & $72.2 \pm 36.4$ & $77.9 \pm 40.5$ & 0.165 \\
Fat intake $(\mathrm{g})$ & $36.6 \pm 26.8$ & $41.3 \pm 31.4$ & 0.120 \\
Dietary fiber intake $(\mathrm{g})$ & $8.1 \pm 4.7$ & $8.6 \pm 6.1$ & 0.353 \\
Calcium intake $(\mathrm{mg})$ & $562.9 \pm 315.6$ & $573.6 \pm 374.7$ & 0.767 \\
Phosphorus intake $(\mathrm{mg})$ & $1,224.2 \pm 522.6$ & $1,301.2 \pm 548.1$ & 0.191 \\
Iron intake $(\mathrm{mg})$ & $16.7 \pm 10.3$ & $17.2 \pm 12.5$ & 0.656 \\
Sodium intake $(\mathrm{mg})$ & $5,496.2 \pm 3,628.9$ & $5,384.9 \pm 3,259.6$ & 0.782 \\
Potassium intake $(\mathrm{mg})$ & $3,221.2 \pm 1,639.4$ & $3,436.9 \pm 1,576.3$ & 0.238 \\
Vitamin A intake $(\mu \mathrm{gg})$ & $746.7 \pm 587.9$ & $936.9 \pm 962.9$ & $0.007^{*}$ \\
Carotene intake $(\mu \mathrm{gg})$ & $3,824.6 \pm 3,276.8$ & $4,886.9 \pm 5,469.9$ & $0.007^{*}$ \\
Retinol intake $(\mu \mathrm{g})$ & $112.1 \pm 229.7$ & $128.1 \pm 568.8$ & 0.600 \\
Vitamin B1 intake $(\mu \mathrm{g})$ & $1.3 \pm 0.7$ & $1.4 \pm 0.8$ & 0.116 \\
Vitamin B2 intake $(\mathrm{mg})$ & $1.2 \pm 0.6$ & $1.3 \pm 0.8$ & $0.020^{*}$ \\
Vitamin B3 intake $(\mathrm{mg})$ & $16.9 \pm 8.8$ & $18.7 \pm 9.8$ & 0.081 \\
Vitamin C intake $(\mathrm{mg})$ & $118.2 \pm 91.6$ & $119.1 \pm 84.4$ & 0.931 \\
\hline P 0.05. PV: & & &
\end{tabular}

*p<0.05. PV: positional vetigo

Table 3. Multivariate binary logistic regression analysis of risk factors for positional vertigo

\begin{tabular}{lccc}
\hline & aOR & $95 \% \mathrm{Cl}$ & $\mathrm{p}$-value \\
\hline Age & 1.029 & $1.006-1.053$ & $0.003^{*}$ \\
Gender ratio $(\mathrm{M}: \mathrm{F})$ & 0.641 & $0.319-1.288$ & 0.251 \\
Serum Hb & 0.996 & $0.993-0.999$ & 0.794 \\
Serum creatinine & 1.013 & $0.771-1.333$ & 0.910 \\
Serum TG & 0.748 & $0.213-2.634$ & 0.064 \\
Carotene intake & 1.000 & $1.000-1.000$ & 0.982 \\
Vitamin A intake & 1.000 & $0.999-1.000$ & 0.523 \\
Vitamin B2 intake & 0.964 & $0.625-1.487$ & 0.594 \\
\hline
\end{tabular}

$* p<0.05$. Hb: hemoglobin, TG: triglyceride, aOR: adjusted odds ratio, $\mathrm{Cl}$ : confidence interval

tion [9], and usually presents as BPPV $[9,10]$. Rare causes include trauma, migraine, and neurovascular compression of the vestibular nerve $[9,11]$. In the present study, PV was diagnosed by questionnaire, otoscopic examination, a hearing test, and the modified Romberg test. However, none of these tests was confirmatory. Dix-Hallpike or roll test data are required to diagnose BPPV. Neurological examination and imaging are employed to examine central causes of vertigo [2].

The prevalence of dizziness and vertigo increase with age $[12,13]$. Jönsson, et al. [12] reported that $30 \%$ of those aged $>70$ years experienced dizziness, as did $50 \%$ of those aged $>85$ years. A German National Telephone Health Interview Survey conducted in 2003 found that the prevalence of BPPV was $3.2 \%$ in females, $1.6 \%$ in males, and $2.4 \%$ overall. The 1 -year incidence was $0.6 \%[3,14]$. In the present survey, age was independently associated with BPPV, which was present in $11 \%$ of those aged $>75$ years, and was significantly more common in females $(p<0.01)$ [14]. Dizziness increased with age in Koreans. The prevalence of dizziness of the past 1 year was reported to be $16.7 \%$. In earlier KNHANES surveys, dizziness was associated with aging, female gender, stress, and HL [13]. We also found that older subjects had a higher prevalence of BPPV, the most common cause of dizziness. Females experienced more dizziness than males [13,14]. We also found a female predominance but this was not statistically significant upon multiple logistic regression.

Our univariate analysis showed that PV was associated with a low level of TG. Low TG levels are associated with a reduced risk of vascular-associated diseases [7]. Low-level TG influences PV development via a mechanism different from that involved in vascular insufficiency. Jönsson, et al. [12] reported that starvation was a risk factor for BPPV. Dietary habits, such as long-duration fasting and skipping breakfast, affect TG levels $[8,15,16]$ and the incidence of BPPV [17]. Dietary habits should be evaluated to clarify the relationship between low TG levels and BPPV development. Additionally, urine ketone levels are elevated during starvation. Although statistical significance was lacking, the urine ketone levels were higher in those with than in those without PV in the current study.

Some studies have reported associations between hyperlipidemia and PV [18-20]. Hyperlipidemia can cause vascular damage to the inner ear $[21,22]$. Labyrinth ischemia can trig- 
Fig. 2. Health-related QoL by PV status. PV was associated with a poor QoL in terms of mobility (A), self-care (B), engagement in usual activities (C), and anxiety/depression (D). QoL: quality of life, $\mathrm{PV}$ : positional vertigo.
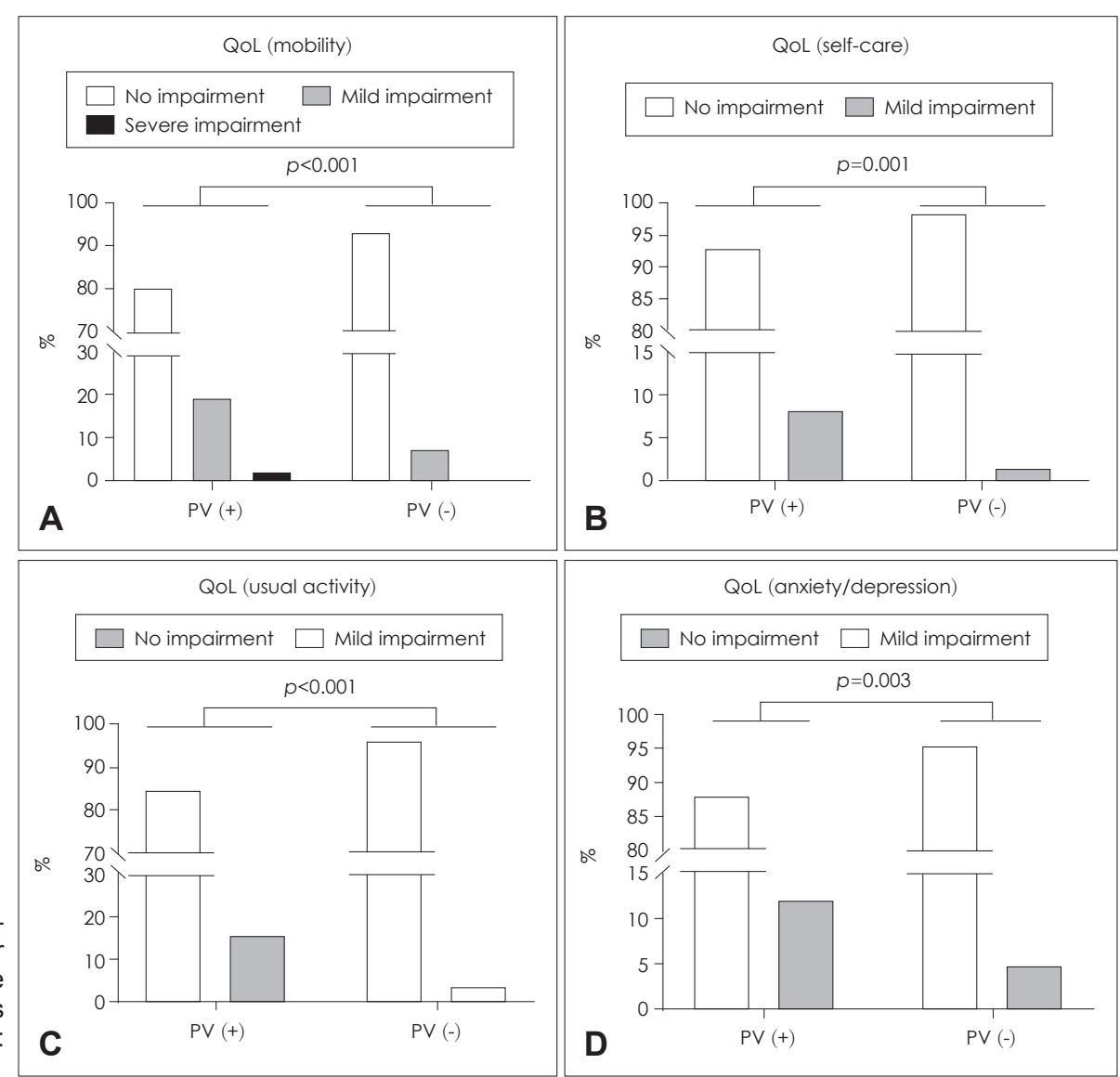

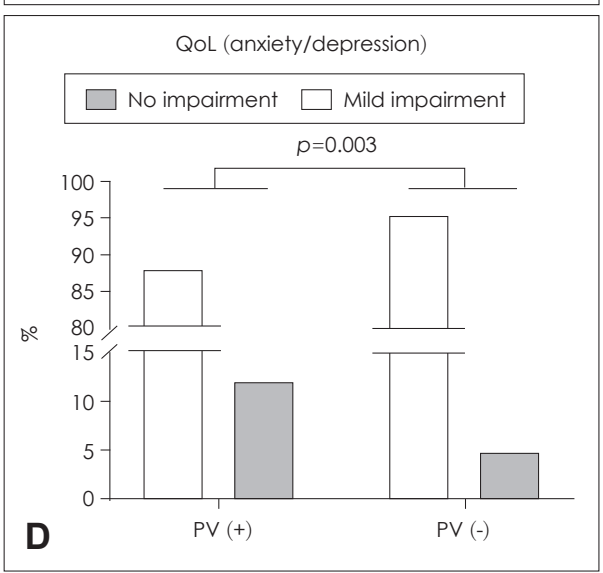

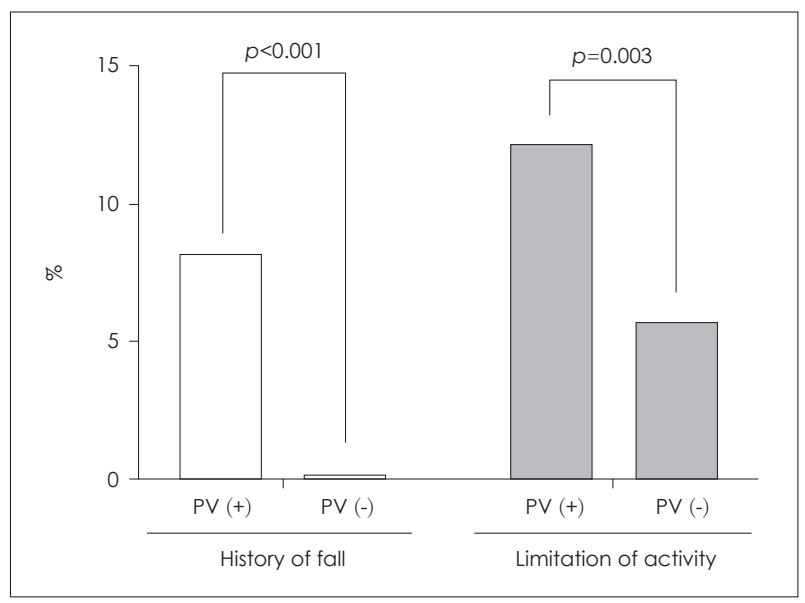

Fig. 3. Fall risks and limitations of daily activity imposed by PV. PV was associated with an increased fall risk $(A)$ and reduced daily activity (B).

ger otoconial detachment from otolith membranes and induce PV [12].

Any association between vitamin D level and BPPV remains controversial. Both vitamin D deficiency and osteoporosis are associated with BPPV [23]. Serum 25-hydroxyvitamin D3 supplementation also reduces BPPV recurrence. However, Karatas, et al. [24] reported that the co-existence of BPPV, os- teoporosis, and vitamin D deficiency was coincidental. Aydin, et al. [25] found that the risks of peripheral vestibular diseases including BPPV were not associated with homocysteine, vitamin B12, or folic acid levels. We did not identify any nutritional or vitamin-associated risk factor.

PV reduces the QoL, directly causing pain and distorting physical, social, emotional, and mental health [26]. Most cases of PV can be cured by appropriate treatment [27]. We found that PV negatively impacted daily life, especially parameters associated with mobility.

PV is associated with a risk of falls and limitations of activity, especially in the elderly $[2,14,28,29]$. Abbott, et al. [29] reported that about half of all patients who fall had PV. We found that the fall risk increased significantly in those with PV. As falls increase the risk of other morbidities including fractures, the elderly should be educated on how to prevent falls during both the acute and resolution stages of PV [2,30]. Additionally, some elderly patients who fall have had hidden PV [28].

Although we analyzed several factors, our study had some limitations. First, as the study design was cross-sectional, we cannot rule out the possibility that reverse causality is at play. Second, many data were subjective (reported by participants), associated with a risk of recall bias. Third, some significant 
parameters are about the same between those with and without PV to apply in clinical setting. However, we did not want to focus on the "value" itself, but find out the tendency. Therefore, meaning of our study was not the cutoff value of significant parameters. Rather, we wanted to show that the lower $\mathrm{Hb}$, $\mathrm{Cr}$, and TG have higher possibility of PV. Finally, although we excluded several confounders, such as stroke and heart and thyroid disease, we may have included other confounders because the study was an epidemiological survey, thus limiting our ability to control for all potential confounders.

In conclusion, age was a risk factor for PV, which negatively affected the QoL, the fall risk, and performance of daily activities. PV should be better managed to increase the QoL and reduce falls, especially in elderly patients.

\section{Acknowledgments}

We thank the Korea Centers for Disease Control and Prevention for providing the data analyzed in the present study. Data Availability Statement: The data used are from the fifth Korean National Health and Nutrition Examination Survey, available via https://knhanes.cdc. go.kr/knhanes/index.do or from Kyungwon Oh at kwoh27@korea.kr. This study was supported by a clinical research grant provided from Seoul National University Boramae Medical Center.

\section{Conflicts of interest}

The authors have no financial conflicts of interest.

\section{REFERENCES}

1) Murdin L, Schilder AG. Epidemiology of balance symptoms and disorders in the community: a systematic review. Otol Neurotol 2015; 36:387-92.

2) Bhattacharyya N, Gubbels SP, Schwartz SR, Edlow JA, El-Kashlan $\mathrm{H}$, Fife $\mathrm{T}$, et al. Clinical pactice guideline: benign paroxysmal positional vertigo (update). Otolaryngol Head Neck Surg 2017;156(3_suppl): S1-47.

3) van Leeuwen RB, Bruintjes TD. Dizziness in the elderly: diagnosing its causes in a multidisciplinary dizziness unit. Ear Nose Throat J 2014;93:162.

4) Furman JM, Cass SP. Benign paroxysmal positional vertigo. N Engl J Med 1999;341:1590-6.

5) Guilemany JM, Martínez P, Prades E, Sañudo I, De España R, Cuchi A. Clinical and epidemiological study of vertigo at an outpatient clinic. Acta Otolaryngol 2004;124:49-52.

6) Corrêa Leite ML, Nicolosi A, Cristina S, Hauser WA, Pugliese P, Nappi G. Dietary and nutritional patterns in an elderly rural population in Northern and Southern Italy: (II). Nutritional profiles associated with food behaviours. Eur J Clin Nutr 2003;57:1522-9.

7) Breier C, Patsch JR, Mühlberger V, Drexel H, Knapp E, Braunsteiner H. Risk factors for coronary artery disease: a study comparing hypercholesterolaemia and hypertriglyceridaemia in angiographically characterized patients. Eur J Clin Invest 1989;19:419-23.

8) Pitha J, Kovář J, Blahová T. Fasting and nonfasting triglycerides in cardiovascular and other diseases. Physiol Res 2015;64 Suppl 3:S32330.

9) Brandt T. Positional and positioning vertigo and nystagmus. J Neurol Sci 1990;95:3-28.

10) Cho EI, White JA. Positional vertigo: as occurs across all age groups. Otolaryngol Clin North Am 2011;44:347-60.

11) Fife TD, Giza C. Posttraumatic vertigo and dizziness. Semin Neurol
2013;33:238-43.

12) Jönsson R, Sixt E, Landahl S, Rosenhall U. Prevalence of dizziness and vertigo in an urban elderly population. J Vestib Res 2004;1:47-52.

13) Koo JW, Chang MY, Woo SY, Kim S, Cho YS. Prevalence of vestibular dysfunction and associated factors in South Korea. BMJ Open 2015;5:e008224.

14) Kollén L, Frändin K, Möller M, Fagevik Olsén M, Möller C. Benign paroxysmal positional vertigo is a common cause of dizziness and unsteadiness in a large population of 75 -year-olds. Aging Clin Exp Res 2012;24:317-23.

15) Hegele RA, Ginsberg HN, Chapman MJ, Nordestgaard BG, Kuivenhoven JA, Averna M, et al. The polygenic nature of hypertriglyceridaemia: implications for definition, diagnosis, and management. Lancet Diabetes Endocrinol 2014;2:655-66.

16) Schultz AR, Neves-Souza RD, Costa Vde S, Meneses-Barriviera $\mathrm{CL}$, Franco PP, Marchiori LL. Is there a possible association between dietary habits and benign paroxysmal positional vertigo in the elderly? The importance of diet and counseling. Int Arch Otorhinolaryngol 2015;19:293-7.

17) Henry CJ, Rivers JP, Payne PR. Protein and energy metabolism in starvation reconsidered. Eur J Clin Nutr 1988;42:543-9.

18) Domínguez-Durán E, Domènech-Vadillo E, Álvarez-Morujo de Sande MG, González-Aguado R, Guerra-Jiménez G, Ramos-Macías Á, et al. Analysis of risk factors influencing the outcome of the Epley maneuver. Eur Arch Otorhinolaryngol 2017;274:3567-76.

19) Rybak LP. Metabolic disorders of the vestibular system. Otolaryngol Head Neck Surg 1995;112:128-32.

20) Duck SW, Prazma J, Bennett PS, Pillsbury HC. Interaction between hypertension and diabetes mellitus in the pathogenesis of sensorineural hearing loss. Laryngoscope 1997;107(12 Pt 1):1596-605.

21) Kimitsuki T. Cholesterol influences potassium currents in inner hair cells isolated from guinea pig cochlea. Auris Nasus Larynx 2017;44: 46-51.

22) Jeong SH, Kim JS, Shin JW, Kim S, Lee H, Lee AY, et al. Decreased serum vitamin $\mathrm{D}$ in idiopathic benign paroxysmal positional vertigo. J Neurol 2013;260:832-8.

23) Talaat HS, Kabel AM, Khaliel LH, Abuhadied G, El-Naga HA, Talaat AS. Reduction of recurrence rate of benign paroxysmal positional vertigo by treatment of severe vitamin D deficiency. Auris Nasus Larynx 2016;43:237-41.

24) Karataş A, Acar Yüceant G, Yüce T, Hacı C, Cebi IT, Salviz M. Association of benign paroxysmal positional vertigo with osteoporosis and vitamin D deficiency: a case controlled study. J Int Adv Otol 2017;13:259-65.

25) Aydin E, Babakurban ST, Ozgirgin ON, Ozlüoğlu LN. The relationship of homocysteine, vitamin B12, folic acid levels with vertigo. Kulak Burun Bogaz Ihtis Derg 2012;22:214-8.

26) Lopez-Escamez JA, Gamiz MJ, Fernandez-Perez A, Gomez-Fiñana M, Sanchez-Canet I. Impact of treatment on health-related quality of life in patients with posterior canal benign paroxysmal positional vertigo. Otol Neurotol 2003;24:637-41.

27) Lopez-Escamez JA, Gamiz MJ, Fernandez-Perez A, Gomez-Fiñana M. Long-term outcome and health-related quality of life in benign paroxysmal positional vertigo. Eur Arch Otorhinolaryngol 2005;262: 507-11.

28) Oghalai JS, Manolidis S, Barth JL, Stewart MG, Jenkins HA. Unrecognized benign paroxysmal positional vertigo in elderly patients. Otolaryngol Head Neck Surg 2000;122:630-4.

29) Abbott J, Tomassen S, Lane L, Bishop K, Thomas N. Assessment for benign paroxysmal positional vertigo in medical patients admitted with falls in a district general hospital. Clin Med (Lond) 2016;16:335-8.

30) Imai $T$, Takeda $N$, Ikezono $T$, Shigeno $K$, Asai M, Watanabe $Y$, et al. Classification, diagnostic criteria and management of benign paroxysmal positional vertigo. Auris Nasus Larynx 2017;44:1-6. 